\title{
Bone marrow mesenchymal stem cells repair the hippocampal neurons and increase the expression of IGF-1 after cardiac arrest in rats
}

\author{
XIAHONG TANG ${ }^{1-3}$, FENG CHEN ${ }^{1-3}$, QINMING LIN ${ }^{1-3}$, YAN YOU $^{1-3}$, JUN KE $^{1-3}$ and SHEN ZHAO $^{1-3}$ \\ ${ }^{1}$ Department of Emergency, Fujian Provincial Hospital, Fujian Medical University; ${ }^{2}$ Fujian Provincial Institute \\ of Emergency Medicine; ${ }^{3}$ Fujian Emergency Medical Center, Fuzhou, Fujian 350009, P.R. China
}

Received December 12, 2016; Accepted August 21, 2017

DOI: $10.3892 /$ etm.2017.5059

\begin{abstract}
The present study aimed to investigate the beneficial effects and underlying mechanisms of bone marrow mesenchymal stem cells (BMSCs) on global ischemic hypoxic brain injury. Cells collected from the femurs and tibias of male Sprague Dawley rats were used to generate BMSCs following three culture passages. A rate model of cardiac arrest (CA) was induced by asphyxia. One hour following return of spontaneous circulation (ROSC), BMSCs were transplanted through injection into the tail vein. Neurological status was assessed using modified neurological severity score (mNSS) tests 1,3 and 7 days following ROSC. Reverse transcription-quantitative polymerase chain reaction (RT-qPCR) and immunohistochemical staining were used to detect insulin-like growth factor 1 (IGF-1) expression in the hippocampus. Furthermore, double-fluorescent labeling of green fluorescent protein (GFP) and IGF-1 was used to detect the IGF-1 expression in transplanted BMSCs. Serum levels of protein S100-B were examined using ELISA. GFP-labeled BMSCs were observed in the hippocampus at 1,3 and 7 days post transplantation through fluorescent microscopy. BMSC transplantation resulted in reduced protein S100-B levels. The mNSS of the BMSC-treatment group was significantly reduced compared with that of the CA group. The RT-qPCR analysis and immunohistochemistry results demonstrated that BMSC treatment significantly increased IGF-1 expression in the hippocampus. In addition, the double-fluorescent labeling results demonstrated that transplanted BMSCs expressed IGF-1 in the hippocampus. The results of the present study suggest that BMSC treatment promotes the recovery of
\end{abstract}

Correspondence to: Dr Feng Chen, Department of Emergency, Fujian Provincial Hospital, Fujian Medical University, 134 East Street, Fuzhou, Fujian 350009, P.R. China

E-mail: cf9066@126.com

Key words: BMSCs, cardiac arrest, global brain ischemia, IGF-1, cerebral resuscitation cerebral function following $\mathrm{CA}$ in rats possibly through the secretion of IGF-1.

\section{Introduction}

Cardiac arrest (CA) was one of the most significant causes of global brain ischemia. Most patients had poor cerebral function after discharge from the emergency room. According to statistics, there were almost 326,200 patients died or disabled from CA (1). However, only 10.6 percent of the emergency patients suffering from CA can survive, and $8.3 \%$ of the patients can retain good neurological function (1). The ischemia brain damage causing by CA was mainly in the hippocampus (2). There were no therapies can treat the behavioral and cognitive dysfunction deriving from the hippocampus damage accompanied by memory loss, emotion change, or even coma, persistent vegetative state and death. Therefore, cerebral protection and cerebral resuscitation are significant after cardiopulmonary resuscitation (CPR).

Because of the complex and diverse mechanisms of brain damage, multi-mechanism treatment is necessary, for example, mild hypothermia therapy. Current updated CPR guidelines recommended that the patients who had out-of-hospital CA with return of spontaneous circulation should have the body cooled to $32-36^{\circ} \mathrm{C}$ for maintenance of $24 \mathrm{~h}$ (3). In general, preliminary study indicated the patients can benefit from induced hypothermia, but the optimal level of hypothermia and the best cooling methods at the beginning of treatment were still under investigation. A number of cerebral protections should be performed by comprehensive treatment, because of the complex mechanisms of brain injury after CA (4). Therefore, the cerebral function may be improved by developing more brain protection plans in cerebral resuscitation study. Stem cell transplant has become important research field of cerebral resuscitation, which can act on several mechanisms of hypoxic-ischemic brain damage (HIBD). The purposes of the study are to explore the protection of bone marrow mesenchymal stem cells (BMSCs) against the global brain injury induced by ischemic and hypoxic and to exam one of its mechanism.

MSCs do not derive from blood forming cells and bone marrow is the most common sources of MSCs (5). Some 
research showed that BMSCs had the following properties: (1) The BMSCs derived from autologous bone marrow are not restricted by ethical issues; (2) Colter and his colleagues (6) discovered that the culturing of $20 \mathrm{ml}$ bone marrow can get $10^{13}$ BMSCs for 3 generations from 6 -week culturing, almost three times more than the original number. Chen et al (7) and Ding et al (8) found that MSCs can repair the nerve in cerebral ischemic animal model; (3) MSCs are able to pass the blood-brain barrier (BBB) to the brain and survive, without the issue of organ rejection (9); (4) Chen et al (10) established the dose-response rat model of intracerebral injection, artery injection and intravenous injection, whose results indicated that neural function could benefit from thef continuous transplantation of BMSCs a month after cerebral injury; (5) Wakabayashi and his colleagues (11) found that nerve injuries and cerebral infarction areas were reduced by MSCs to the rat model of middle cerebral artery occlusion (MCAO), and the transplantation especially increased the insulin-like growth factor 1 (IGF-1) secretion.

In this research, CA was induced by asphyxia. One hour after ROSC, BMSCs were transplanted by injecting into the vein of the tails. Observing the effects of BMSCs on nerve function of rats with $\mathrm{CA}$, this paper discussed the neuroprotection of global cerebral ischemia and the mechanisms involved.

\section{Materials and methods}

Experimental animals. Primary cells were cultured from the specific pathogen-free (SPF) male healthy Sprague-Dawley (SD) rats weighing 100-110 g, and the SPF male healthy SD rats weighing 250-350 g were used to established rat model. The rats fasted except water the night before experimental operation. All the rats were provided by Center for Animal Testing of Beijing Vital River. Animal permit no. SCXK (Jing) 2012-0001. All procedures were according to the Guidance Suggestion of Caring Laboratory Animals (2006. 09. 30).

Isolation and culture of BMSCs. The young SD rats weighing 100-110 g were sacrificed, and femurs and tibias were isolated from the muscle in the laminar flow cabinet (Suzhou Antai Airtech Co., Ltd., Suzhou, China). The bone cavity was repeatedly rinsed with Dulbecco's modified Eagle's medium/Ham's F12 (DMEM/F12) without fetal bovine serum (FBS) (both from Hyclone, Logan, UT, USA). The washing fluid was put into centrifuge tube, and the supernatant was discarded after centrifuging at $1,500 \mathrm{rpm}$ for $5 \mathrm{~min}$. The precipitation was mixed by $8 \mathrm{ml}$ DMEM/F12 with $10 \% \mathrm{FBS}$, divided into $25 \mathrm{~cm}^{2}$ culture flasks, and incubated in a Tri-Gas incubator (Sanyo, Osaka, Japan). Half of the medium was changed after $24 \mathrm{~h}$; the whole medium was changed after $48 \mathrm{~h}$; and the medium was changed every other day.

The cells were trypsinized by $2 \mathrm{ml} 0.25 \%$ pancreatin (Gibco; Thermo Fisher Scientific, Inc., Waltham, MA, USA) containing $0.1 \mathrm{mM}$ ethylenediaminetetraacetic acid (EDTA) for 2 min. Cell passaged (1:2) when the cells fusion reach $90 \%$, and subculture was repeated when $90 \%$ of cells fused. The P3 generation cells were used for further testing and transplantation. As the paper described above $(12,13)$, BMSCs were measured by flow cytometry analysis of CD 29, CD 90, CD 45 and CD 11b (BioLegend, Inc., San Diego, CA, USA), and the expressions of CD 29 and CD 90 were 97.15 and $99.02 \%$. The expressions of CD 45 and CD $11 \mathrm{~b}$ were 0.36 and $1.45 \%$. It confirmed that BMSCs had been successfully isolated and cultured, which can be used for further research.

Prior to transplantation, the BMSCs were collected at P3 generation of growth, washed three times by phosphatebuffered solution (PBS; Hyclone), and incubated with $10 \mu \mathrm{l}$ of $1 \times 10^{8} \mathrm{TU} / \mathrm{ml}$ green fluorescent protein (GFP; Shanghai GenePharma Co., Ltd., Shanghai, China). The medium was changed after $24 \mathrm{~h}$, and we observed the GFP under the fluorescence microscope after $72 \mathrm{~h}$. The GFP was transfected into the BMSCs higher than $80 \%$ and can be used for further experiments.

Rat model of CPR. Adult male SD rats weighing 250-350 g were selected and divided into three groups randomly: sham operation group $(n=15)$, CA group $(n=15)$ and BMSCs treatment group $(n=18)$, we made same models of asphyxia-induced CA. The 1st day, the 3rd day and the 7 th day were chosen as the time-points to observe after ROSC. One hour after ROSC, $\sim 1 \times 10^{6}$ BMSCs were transplanted by injecting into the vein of the tails. There were 6 rats on each time-point in BMSCs treatment group, and 5 rats in the other one.

The adult rats were anesthetized by intraperitoneal injection $4.5 \mathrm{mg} / 100 \mathrm{~g}$ pentobarbital solution. The skin of the neck, chest and groin was prepared before the rats' incisors were fixed on the operation table in supine position. Endotracheal intubation was performed by shining a flashlight penetrating through the neck skin. The tracheal tube of 14 gauge cannula (Becton Dickinson, Franklin Lakes, NJ, USA) was fixed to the jaw with suture after the confirmation of successful intubation. In anesthetic condition, right femoral artery was separated, and the blood flow was occluded by ophthalmic forceps. The distal artery was ligated; the proximal artery was cut a small slit by ophthalmic scissors; and then a heparinized polyethylene 50 (PE-50) catheter was advanced into the femoral artery for measurement of mean arterial pressure (MAP) before the pipe was fixed. Map was measured by BL-420 biological functional system (Chengdu Taimeng Technology Co., Ltd., Chengdu, China). Electrocardiogram (ECG) was also monitored by the system (lead II).

The physiological baseline parameters were recorded when the rats was totally conscious. $0.05 \mathrm{mg} / 100 \mathrm{~g}$ vecuronium was injected into three-way tube connected to the PE-50 catheter, and the tracheal tube was blocked by a syringe. The standard of CA was the MAP $\leq 25 \mathrm{mmHg}$, and the artery pulse wave without fluctuation (14). After 5 min of CA, the syringe was withdrawn from tracheal tube, and the freehand external cardiac compression begun immediately with mechanical ventilation with $\mathrm{FiO}_{2}$ of $100 \%$ by the rodent ventilator (Shanghai Alcott Science and Technology Co., Ltd.). The tidal volume was $0.6 \mathrm{ml} / 100 \mathrm{~g}$ and the breathing rate was $100 \mathrm{bpm}$. The compression/ventilation ratio was $2: 1$. The depth of compression was $1 / 3$ of anteroposterior chest diameter, or adjusted by the change of MAP. The $0.1 \mathrm{ml}$ adrenal was injected into three-way tube after 2 min of compression, with the same dose every $2 \mathrm{~min}$. The criterion of ROSC was returned of supraventricular rhythm with the MAP $\geq 60 \mathrm{mmHg}$ lasting for at least $10 \mathrm{~min}$ (15). 
Table I. Modified neurological severity scores.

Items

\section{Motor tests}

Raising rat by tail

Flexion of forelimb

Flexion of hindlimb

Head moved $>10$ to vertical axis within 30

Placing rat on the floor (normal, 0 ; maximum, 3)

Normal walk

Inability to walk straight

Circling toward the paretic side

Fall down to the paretic side

Sensory tests

Visual and tactile placing

Proprioceptive test (deep sensory)

Beam balance tests

Grasps side of beam

Hugs the beam and one limb falls down from the beam

Hugs the beam and two limb fall down from the beam, or spin on beam ( $>60 \mathrm{sec})$

Attempt to balance on the beam but fall off ( $>40 \mathrm{sec})$

Attempt to balance on the beam but fall off ( $>20 \mathrm{sec}$ )

Fall off with no attempt to balance or hand on to the beam

Reflexes (blunt or sharp stimulation) absent of :

Pinna reflex (a head shake when touching the auditory meatus)

Corneal reflex (an eye blink when lightly touching the cornea

with cotton

Startle reflex (a motor response to a brief loud paper noise)

Seizures, myoclonus, myodystony

Maximum points

Score: $13-18$ severe; 7-12 moderate; 1-6 mild injury.

Table II. The primers and GAPDH.

\begin{tabular}{llc}
\hline Primer name & Sequence $\left(5^{\prime}-3^{\prime}\right)$ & Length $(\mathrm{bp})$ \\
\hline rat-GAPDH-forward & AGTTCAACGGCACAGTCAAGG & 121 \\
rat-GAPDH-reverse & ACATACTCAGCACCAGCATCAC & 156 \\
rat-IGF1-forward & CTGGTGGACGCTCTTCAGTTC & 15 \\
rat-IGF1-reverse & ACAGTACATCTCCAGCCTCCTC & \\
\hline
\end{tabular}

GAPDH, glyceraldehyde-3-phosphate dehydrogenase; IGF1, Insulin-like growth factor 1.

The 5-15 $\mu \mathrm{g} / \mathrm{kg}$.min dopamine was injected when the blood pressure dropped. If there was no ROSC after $10 \mathrm{~min}$ of chest compression, the rat was to be considered as recovery failure. Mechanical ventilation was continued for $30 \mathrm{~min}$ after ROSC, and continuously recorded for ECG and MAP. The sham operation group rats underwent the same operation except the CA. All the operation was done by the same experimenter.
Transplantation of BMSCs. One hour after ROSC, in BMSCs treatment group, $\sim 1 \times 10^{6}$ GFP-labeled BMSCs were prepared for transplantation. The cells were resuspended in $0.5 \mathrm{ml}$ PBS for transplantation by injecting into the vein of the tails. After injecting, the catheter and the cannula were removed with the femoral artery ligated. 8 million IU of penicillin was injected by intraperitoneal injection. The rats were totally awake and returned to the animal cages. Incandescents were used for 
Table III. Thermocycle features for polymerase chain reaction.

\begin{tabular}{lcc}
\hline Temperature & Time (sec) & Cycles \\
\hline $94^{\circ} \mathrm{C}$ (pre-degeneration) & 30 & $\begin{array}{c}\text { Stage } 1 \\
1 \text { cycle } \\
\text { Stage } 2\end{array}$ \\
$95^{\circ} \mathrm{C}$ (denature) & 5 & 40 cycles \\
& & \\
$61^{\circ} \mathrm{C}$ (primer annealing) & 30 & Melting curve \\
$72^{\circ} \mathrm{C}$ (extension) & 30 & 1 cycle \\
$95^{\circ} \mathrm{C}$ & 15 & \\
$55^{\circ} \mathrm{C}$ & & \\
$95^{\circ} \mathrm{C}$ & 15 & 15
\end{tabular}

heating. Rats were fed by $10 \%$ glucose injection on the first day after ROSC, hereafter, fed by routine feed.

Nerve function defect grade in rats. The neurological function of rats after ROSC at 1st day, the 3rd day and the 7th day was assessed by the modified neurological severity scores (mNSS; Table I), which can evaluate the extent of neurological damage in motor, sensory, balance and reflex. The more the scores, the more serious nerve injury (16).

Serum levels of $S 100 B$. The rats at day 1, 3, 7 were anesthetized by intraperitoneal injection $4.5 \mathrm{mg} / 100 \mathrm{~g}$ pentobarbital solution. Thoracic cavity was opened to expose the heart, and the $5 \mathrm{ml}$ blood was taken from the right ventricle by a needle. The supernatant kept under $-80^{\circ} \mathrm{C}$ after centrifuging at 3,000 rpm for $15 \mathrm{~min}$. Serum levels of S100B were examined by enzyme-linked immunosorbent assay (ELISA) kit (NeoBioscience, Shenzhen, China) following the instructions.

Tissue sampling and slices. The skull was opened quickly after blood collection by the steps. The left hippocampus was removed and placed in the $4 \%$ paraformaldehyde rapidly, and immersed for 3 days, and then the frozen sections were made $20 \mu \mathrm{m}$ after dehydration. The right hippocampus was placed in sterile tube, and stored in the liquid nitrogen jar for real-time quantitative PCR.

Real-time quantitative PCR analysis. Total RNA from 48 of right hippocampus samples were extracted with TRIzol reagent (Qiagen GmbH, Hilden, Germany). Primers were designed by Shanghai Sunny Biotechnology Co., Ltd. (Shanghai, China). Reverse transcription was performed by PrimeScript RT Master Mix (Takara Bio, Inc., Otsu, Japan). Real-time quantitative PCR was performed by ECO fluorogenic quantitative PCR (Illumina, Inc., San Diego, CA, USA) using fluorescent quantitative reagent kit (Takara Bio, Inc.). Total two primers were designed by Genergy Biotechnology (Shanghai) Co., Ltd. (Shanghai, P.R. China). (Table II).

The solution of reverse transcription was centrifuged slightly, and the reverse transcription was reacted at $37^{\circ} \mathrm{C}$ for $15 \mathrm{~min}$, and then reverse transcriptase was inactivated for $30 \mathrm{sec}$. The cDNA was stored at $-20^{\circ} \mathrm{C}$, and it was diluted

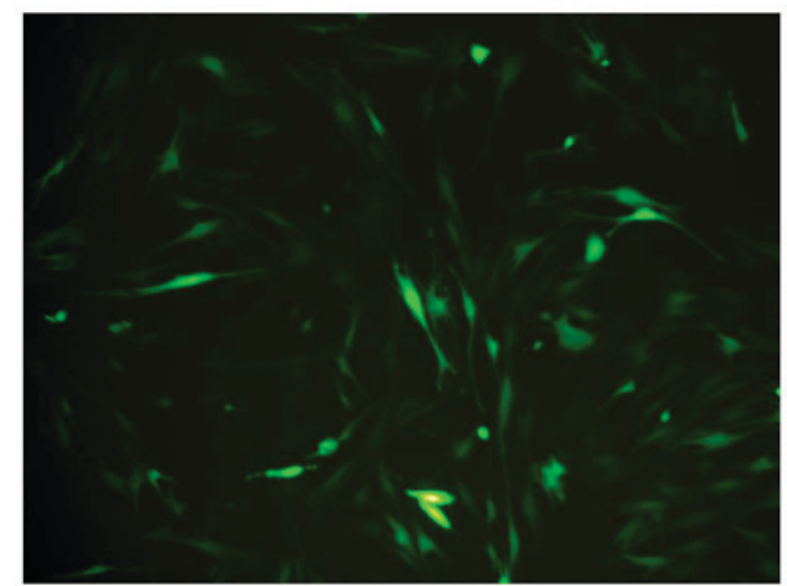

Figure 1. Bone marrow mesenchymal stem cells labeled with green fluorescent protein $72 \mathrm{~h}$ after transfection (magnification, $\mathrm{x} 200$ ).

10 times in this experiment. The parameters of thermal cycle of PCR were showed in the Table III. The expression of mRNA were calculated based on $2^{-\Delta \Delta \mathrm{ct}}$ method (17).

Detection of the expression of IGF-1 in BMSCs by the double fluorescent labeling of GFP and IGF-1. Before the sections were covering with citric acid buffer $(\mathrm{pH} 6.0)$, the frozen sections were washed in PBS for 5 min three times, and the sections were washed in PBS again. The tissue samples were blocked by normal serum (Abcam, Cambridge, UK ) a $37^{\circ} \mathrm{C}$ for $1 \mathrm{~h}$. The IGF-1 antibody (Novus Biologicals, Littleton, CO, USA) was added according to the manufacturer's directions, after the normal serum was sop by filter. The tissue samples were washed in PBS for 5 min three times, before covered by diluted secondary antibody (Abcam), incubated at $37^{\circ} \mathrm{C}$ for $40 \mathrm{~min}$, and then washed in PBS for $5 \mathrm{~min}$ three times. After added the DAPI $(1: 1,000)$ for $10 \mathrm{~min}$, the samples were washed in PBS for 5 min three times immediately, and dehydrated and mounted with neutral gum.

Statistical analysis. Measurement data are showed as means \pm standard deviations, and the IBM SPSS 19.0 was used for statistical analysis. One-way ANOVA was performed for the statistical method, the homogeneity of variance and analysis of variance were compared in every group. Measurement data were compared with S-N-K method, and statistical significance was determined at $\mathrm{P}<0.05$.

\section{Results}

Observation of GFP-labeled BMSCs. BMSCs were spindle shaped as usual, after marked by BMSCs. The growth, proliferation and activity of BMSCs were not influenced by GFP. After 3 days, The BMSCs appeared fluorescent green under a fluorescent microscope. The transfection efficiency was greater than $80 \%$ of the BMSCs, and it showed GFP can perform as the biomarker of BMSCs (Fig. 1).

Nerve functional score in rats. Before the experimental operation, the score in rats of all groups was zero, and there were no significant differences $(\mathrm{P}>0.05)$. The $\mathrm{mNSS}$ score of $\mathrm{CA}$ 


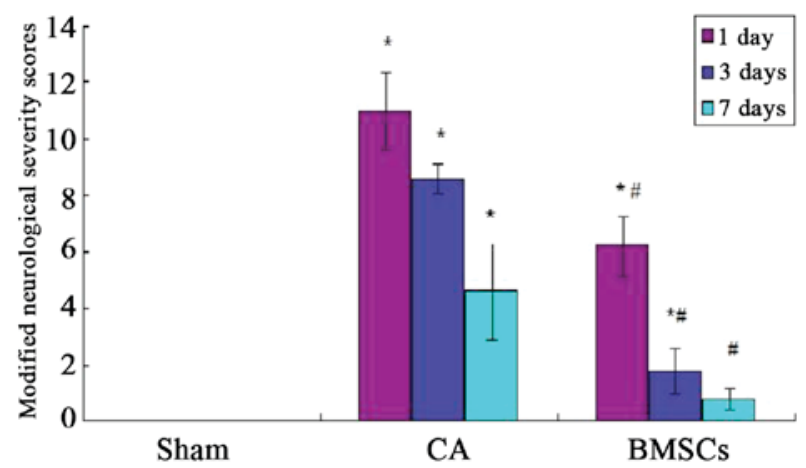

Figure 2. Modified neurological severity scores in rats. " $\mathrm{P}<0.05$ vs. sham operation group; ${ }^{*} \mathrm{P}<0.05$ vs. cardiac arrest group. $\mathrm{CA}$, cardiac arrest; BMSCs, bone marrow mesenchymal stem cells.

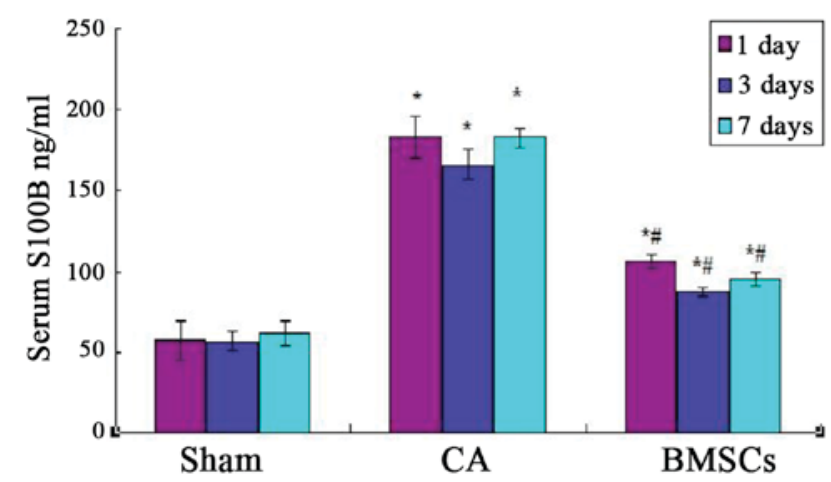

Figure 3. Levels of S100B in rats. ${ }^{~} \mathrm{P}<0.05$ vs. sham operation group; ${ }^{~} \mathrm{P}<0.05$ vs. cardiac arrest group. CA, cardiac arrest; BMSCs, bone marrow mesenchymal stem cells.

group was higher than sham operation group significantly after ROSC at 1 st, 3rd and 7th day $(\mathrm{P}<0.05)$; the mNSS score of BMSCs treatment group was reduced significantly $(\mathrm{P}<0.05)$. However, compared with sham operation group, the mNSS score of BMSCs treatment group was no statistical differences at 7 th day $(\mathrm{P}>0.05$; Fig. 2$)$.

Serum levels of S100B. As shown in Fig. 3, Serum levels of S100B of cell-transplanted group and CA group were higher than sham operation group and CA group significantly after ROSC at each time point. Cell-transplanted group decreased significantly after ROSC at the 1st, 3rd, and 7th day than CA group at the content of S100B. The standard curve of serum S100B levels was showed in Fig. 4.

Detection of the expression of IGF-1 in BMSCs by the double fluorescent labeling of GFP and IGF-1. The GFP-labeled BMSCs can be detected in hippocampus under a fluorescent microscope (Olympus, Tokyo, Japan) at day 1,3 and 7. The red stain was the IGF-1; GFP was observed green; and the cell nucleus was blue after DAPI dyeing. The cells located in hippocampus were appeared green and blue. The BMSCs secreted IGF-1 were green dyeing and red dyeing. The proportion of positive cells double stained by IGF-1 and GFP, and also labeled by DAPI were $6.89,7.44,7.26 \%$ at 1 st day, 3rd and 7th day, respectively (Figs. 4-6).
A

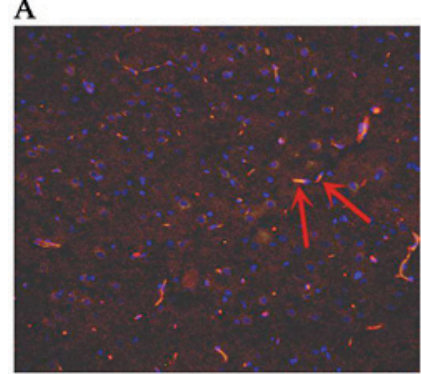

B
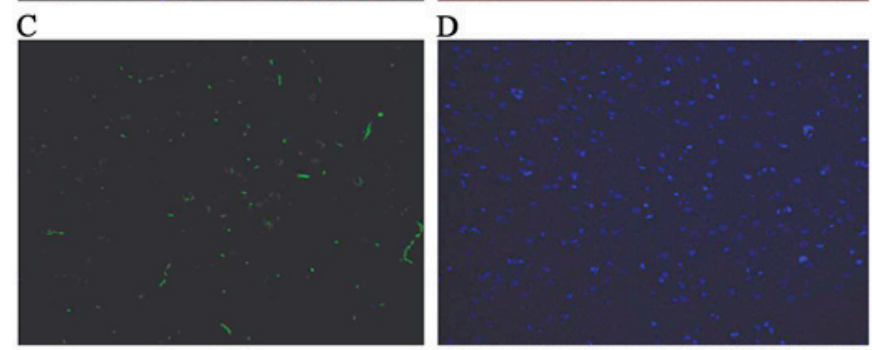

Figure 4. Fluorescence in hippocampus at day 1 after ROSC. The arrows indicate IGF-1-positive bone marrow mesenchymal stem cells. (A) Green and red staining to detect positive cells; (B) red staining to detect IGF-1; (C) green staining to detect green fluorescent protein; (D) blue staining to detect the cell nucleus. Magnification, x200. ROSC, return of spontaneous circulation; IGF-1, insulin-like growth factor 1.
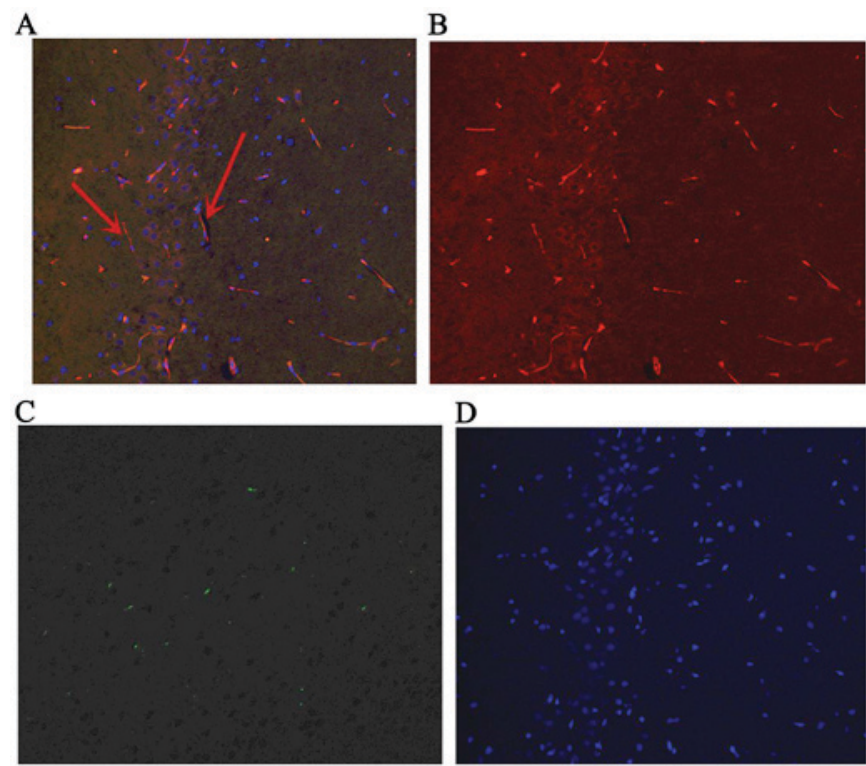

D

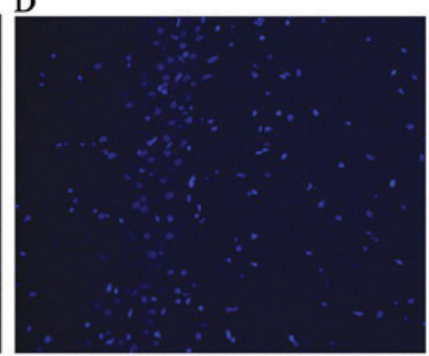

Figure 5. Fluorescence in hippocampus at day 3 after ROSC. The arrows indicate IGF-1-positive bone marrow mesenchymal stem cells. (A) Green and red staining to detect positive cells; (B) red staining to detect IGF-1; (C) green staining to detect green fluorescent protein; (D) blue staining to detect the cell nucleus. Magnification, x200. ROSC, return of spontaneous circulation; IGF-1, insulin-like growth factor 1 .

Quantitative PCR assays for IGF-1 in the hippocampus. Real-time quantitative PCR analysis showed that there was basic expression of IGF-1 in sham group. The results confirmed IGF-1 mRNA relative amount of BMSCs treatment group was significantly higher than that of sham operation group and CA group at each time point. The expression of IGF-1 mRNA in CA group was significantly increased compared with sham group at the 1st and 3rd day. There was no statistical difference of the expression of IGF-1 

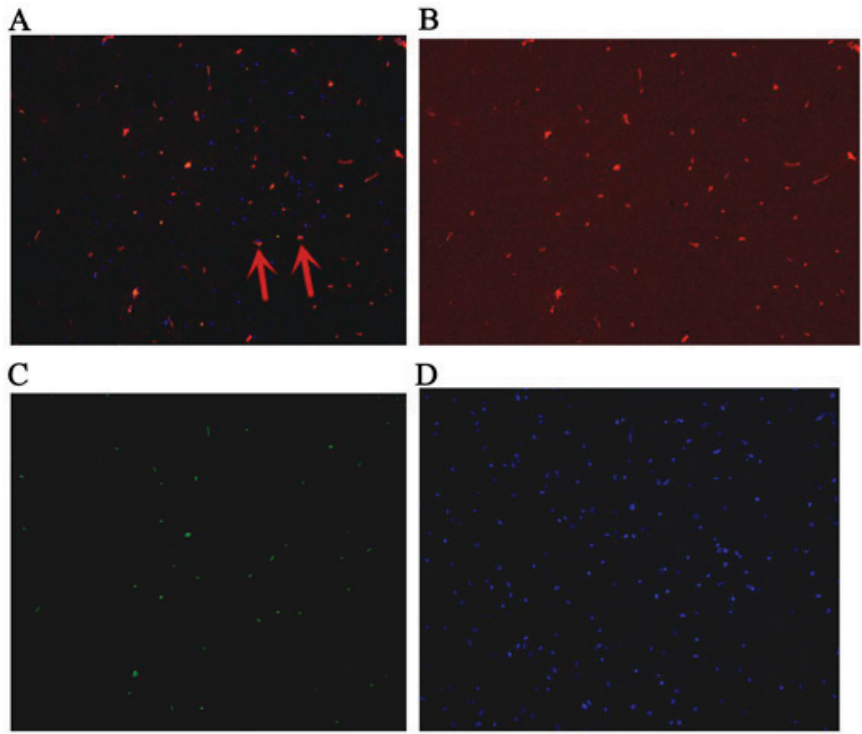

Figure 6. Fluorescence in hippocampus at day 7 after ROSC. The arrows indicate IGF-1-positive bone marrow mesenchymal stem cells. (A) Green and red staining to detect positive cells; (B) red staining to detect IGF-1; (C) green staining to detect green fluorescent protein; (D) blue staining to detect the cell nucleus. Magnification, x200. ROSC, return of spontaneous circulation; IGF-1, insulin-like growth factor 1.

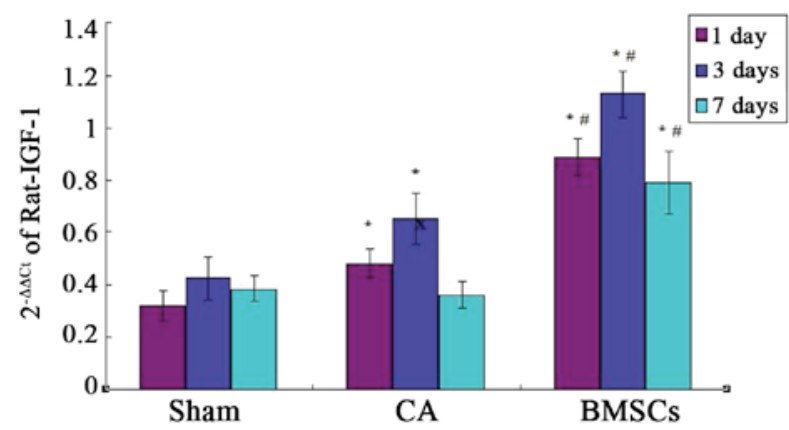

Figure 7. The expression of IGF-1 mRNA in sham, CA, BMSCs-treated groups ${ }^{*} \mathrm{P}<0.05$ vs. sham operation group; ${ }^{\#} \mathrm{P}<0.05$ vs. cardiac arrest group. IGF-1, insulin-like growth factor 1; CA, cardiac arrest; BMSCs, bone marrow mesenchymal stem cells.

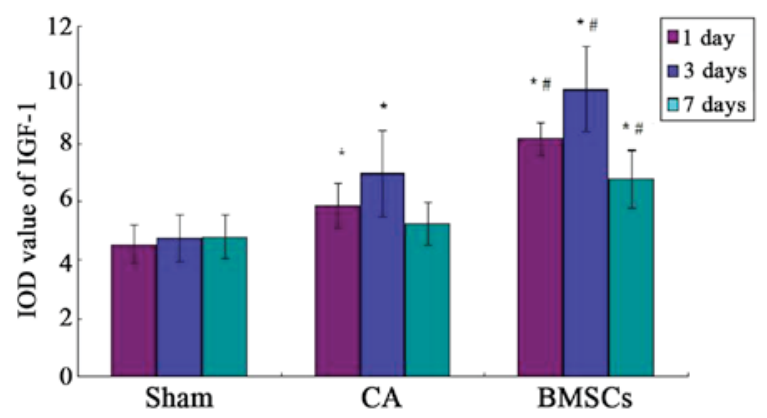

Figure 8. Comparison of the IOD value of IGF-1 in different groups. ${ }^{*} \mathrm{P}<0.05$ vs. sham operation group; ${ }^{\#} \mathrm{P}<0.05$ vs. cardiac arrest group. IOD, integrated optical density; IGF-1, insulin-like growth factor 1; CA, cardiac arrest; BMSCs, bone marrow mesenchymal stem cells.

mRNA between CA group and sham group at day 7, as shown in Fig. 7.
Observation of the expression of IGF-1 in hippocampus by immunohistochemistry. The IGF-1 positive-staining cells appeared light brown dyeing. A few light brown dyeing can be detected in sham group, which showed IGF-1 was weakly expressed in the hippocampus of the rat.Immunohistochemistry results demonstrated the hippocampus expressed IGF-1 positive, which were found in cell-transplanted group. There was a little expression in CA group, both groups were higher than that of sham operation group. But the expression in CA group had no statistical difference with sham operation group at 7 th day, as shown in Figs. 8-11.

\section{Discussion}

BMSCs are pluripotent, low- immunogenic, can be obtained easily and convenient (18). BMSCs which originate from bone marrow, have the ability to self-renew, multi-differentiating, nerve repair as well as to promote the nerve cells to proliferation. This method is widely used in treating nervous system diseases. The present studies on the effects of BMSCs repairing the nerve damage after global ischemia induced by CA were still comparatively little. Previous showed that the neuron loss and the apoptosis of neurons in the hippocampus were improved in the BMSCs-treated group compared to the CA group $(19,20)$. These researches proved that global ischemia can be improved by BMSCs transplantation. In this study, the GFP-labeled BMSCs were distributed in the hippocampus which was easily damaged after CA. It illustrated BMSCs had the ability to pass the BBB and migrated to the lesion. The rats' neurological status was assessed by mNS tests. The more the scores, the more serious the nerve injury was. Our results showed that the abilities of motor, sensory, balance and reflex were improved in the BMSCs treated group, compared to the CA group. There was a number of behavior function damage in rats after CA with the symptoms of twitching, convulsions, low spirits and anorexia. The damaged neurological function had limited recovery. Some researches shown that the Nogo-A secreted by oligodendrocytes and glial scar formed by astrocytes can suppress the growth of axon and myelination $(21,22)$. The expression of Nogo-A can be decreased by the transplantation of BMSCs, and the time of reduction of scar was reduced (23).

The S100B were distributed in the central nevous system and play a vital role in determining cell proliferation, differentiation, nutrition and metabolism. If the brain tissue, neurons or blood-brain barrier was damaged, the over-expressed S100B were released into the blood. Researches found a positive correlation between the levels of S100B and the damage degree of injury brain, and serum levels of S100B can be an objective measure of brain damage after CPR. The results of the study indicate that BMSCs can effective reduce the levels of S100B and improve nerve damage after cerebral resuscitation.

The mechanisms of BMSCs in brain resuscitation included cell differentiation, immune regulation, promotion of angiogenesis, anti-apoptosis and secretion of neurotrophic factors. A study indicated that BMSCs can secrete brain-derived growth factor (BDGF), nerve growth factor (NGF), basic fibroblast growth factor (bFGF), hepatocyte growth factor (HGF), vascular endothelial growth factor 
A

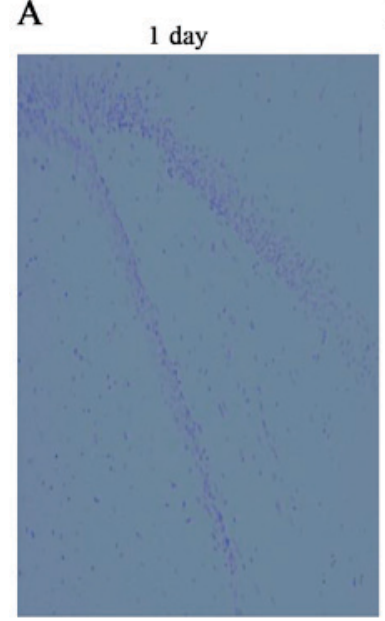

B

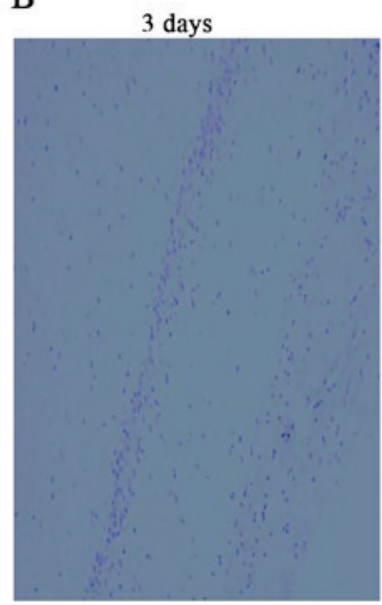

$\mathrm{C}$

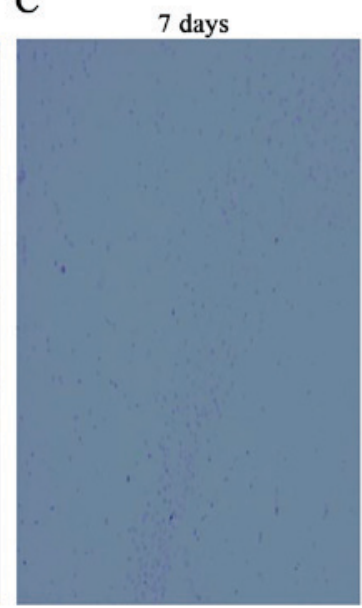

Figure 9. Results of insulin-like growth factor 1 by immunohistochemistry in the hippocampus in the sham group at days (A) 1 , (B) 3 and (C) 7 .

A

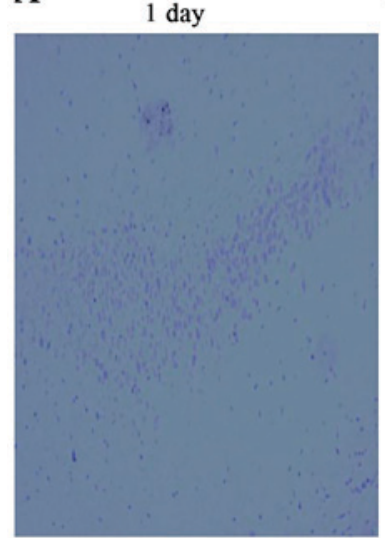

B

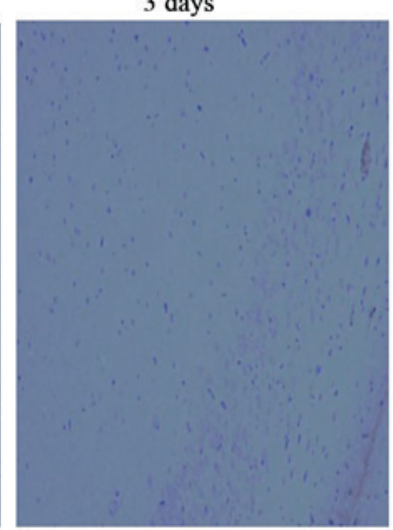

$\mathrm{C}$

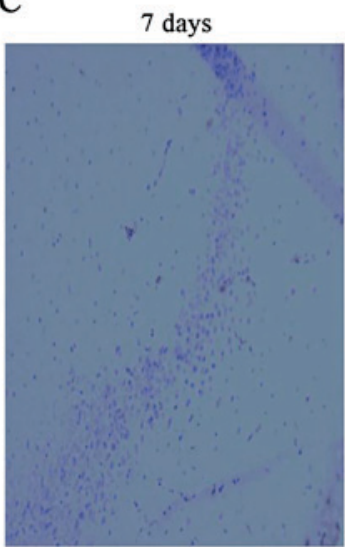

Figure 10. Results of insulin-like growth factor 1 by immunohistochemistry in the hippocampus in the cardiac arrest group at days (A) 1 , (B) 3 and (C) 7 .

A

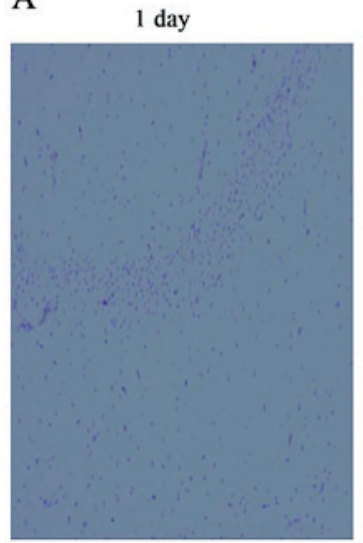

B

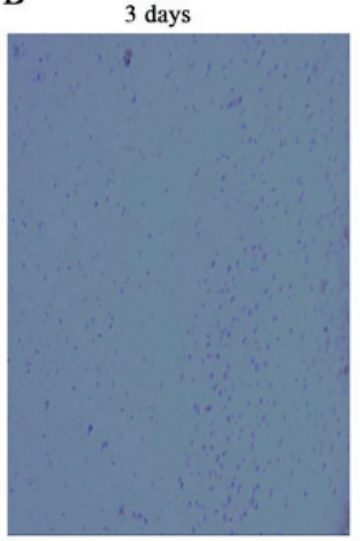

$\mathrm{C}$

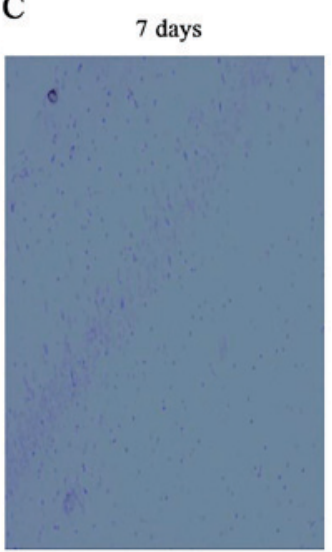

Figure 11. Results of insulin-like growth factor 1 by immunohistochemistry in the hippocampus in the bone marrow mesenchymal stem cell-treated group at days (A) 1 , (B) 3 and (C) 7 .

(VEGF) and IGF-1 (24). A study shown that the growth of axons was promoted by hippocampal cells co-culturing with BMSCs, and the cell apoptosis was suppressed; the results of proteome analysis and ELISA demonstrated IGF-1 existed in the culture medium, and the DNA sequencing analysis also indicated the high expression of IGF-1 in neurons (25). Research shown that the hippocampus and the learning ability were improved in the neonatal rats of hypoxic-ischemic injury with the secretion of IGF-1, after the adipose tissue-derived stem cells were transplanted 
via jugular vein. Moreover, the nerve injuries and cerebral infarction areas were reduced by the transplantation of MSCs to the rat model of MCAO, and the secretion of IGF-1 was increased. In this experiment, the result of the double fluorescent labeling of GFP and IGF-1 showed that the IGF-1-secreting BMSCs marked by DAPI were green dyed and red dyed, respectively, which indicated that BMSCs can home to the lesion in the hippocampus and survived to secrete IGF-1.

Insulin-like growth factor-1, a 70-amino-acid polypeptide, named for the homology with insulin belongs to the tyrosine kinase receptor $\mathrm{E}$ and regulated by IGF-1 binding proteins (IGFBPs). IGF-1 mRNA expresses in the tissues of muscle, liver and nerves, worked via the pathway of endocrine, autocrine and paracrine (26). Study had found that the expression of IGF-1 mRNA was increased on local cerebral ischemia, and the expression quantity was proportional to the brain injury extent (27). Lehtinen et al (28) argued that IGF-1 was good for the differentiation of the neural cells, axonal connections, accumulation of contactin and the sodium channel open. A study confirmed IGF-1 had the protective effect on the HIBD (29). Previous studies showed that IGF-1 can enhance the activity of BDNF and VEGF $(30,31)$, and there were no growth factors can enhance the activity of IGF-1. Peripherally administered IGF-1 is not easily to pass BBB because of its big molecular weight, and direct injection to brain can cause twice trauma, so the clinical application of IGF-1 is limited (32). However, BMSCs can secrete the IGF-1 homing to the ischemia areas, and it provides an appropriate administration route to avoid BBB.

In this study, the expression of IGF-1 mRNA in the CA group was significantly increased compared with that of the sham group, which confirmed the expression of IGF-1 in the model of CA induced by asphyxia was similar to the model of focal cerebral ischemia, and have a same tendency of IGF-1 mRNA. The results of double fluorescent labeling confirmed BMSCs can definitely promote the secretion of IGF-1 in the rats of CA induced by asphyxia. We found that the expression in the CA group had no statistical difference with the sham operation group at 7th day, but the expression in the BMSCs-treated group increased significantly compared to the other groups. Combining the results of the mNSS test, the mNSS score of the BMSCs treatment group had no statistical differences at 7th day, which illustrated BMSCs can prolonge the time of expression result in the improvement of neurological function.

However, this experiment also showed some limitations. One limitation was that the study did not illustrate the IGF-1 was a factor in improving cerebral resuscitation after transplantation of BMSCs. The further research was planning to block IGF-1 in rats performed by IGF-1 antibody or siRNA technology. Another limitation was the mechanisms of brain protection of IGF-1 were not explained. The mechanisms of IGF-1 were still in research. Possible mechanisms of IGF-1 included: i) It can inhibit the apoptosis of the nerve cells (33-35); ii) it can resist the function of excitatory amino acid (36); iii) It prevents the calcium overload after cerebral ischemia reperfusion (35). (4) It can decrease the cerebral vascular resistance and improve blood circulation (37).
In summary, the hippocampal neuron in rats after CPR can benefit from BMSCs treatment possibly via secreted IGF-1.

\section{Acknowledgements}

This study was funded by National Key Clinical Specialty Construction Project of China (Emergency Medicine 2012) and Fujian Provincial Natural Fund Subject (2014J01289).

\section{References}

1. Writing Group Members; Mozaffarian D, Benjamin EJ, Go AS, Arnett DK, Blaha MJ, Cushman M, Das SR, de Ferranti S, Després JP, et al: Heart Disease and Stroke Statistics-2016 Update A Report From the American Heart Association. Circulation 133: E38-E360, 2016.

2. Popp E, Vogel P, Teschendorf P and Böttiger BW: Effects of the application of erythropoietin on cerebral recovery after cardiac arrest in rats. Resuscitation 74: 344-351, 2007.

3. Callaway CW, Donnino MW, Fink EL, Geocadin RG, Golan E, Kern KB, Leary M, Meurer WJ, Peberdy MA, Thompson TM and Zimmerman JL: Part 8: Post-cardiac arrest care: 2015 American heart association guidelines update for cardiopulmonary resuscitation and emergency cardiovascular car. Circulation 132 (18 Suppl 2): S465-S482, 2015.

4. Teschendorf P, Vogel P, Wippel A, Krumnikl JJ, Spöhr F, Böttiger BW and Popp E: The effect of intracerebroventricular application of the caspase-3 inhibitor zDEVD-FMK on neurological outcome and neuronal cell death after global cerebral ischaemia due to cardiac arrest in rats. Resuscitation 78: 85-91, 2008.

5. Mohal JS, Tailor HD and Khan WS: Sources of adult mesenchymal stem cells and their applicability for musculoskeletal applications. Curr Stem Cell Res Ther 7: 103-109, 2012.

6. Colter DC, Class R, DiGirolamo CM and Prockop DJ: Rapid expansion of recycling stem cells in cultures of plastic-adherent cells from human bone marrow. Proc Natl Acad Sci USA 97: 3213-3218, 2000.

7. Chen A, Siow B, Blamire AM, Lako M and Clowry GJ: Transplantation of magnetically labeled mesenchymal stem cells in a model of perinatal brain injury. Stem Cell Res 5: 255-266, 2010.

8. Ding X, Li Y, Liu Z, Zhang J, Cui Y, Chen X and Chopp M: The sonic hedgehog pathway mediates brain plasticity and subsequent functional recovery after bone marrow stromal cell treatment of stroke in mice. J Cereb Blood Flow Metab 33: 1015-1024, 2013.

9. Kim SJ, Moon GJ, Chang WH, Kim YH and Bang OY; STARTING-2 (STem cell Application Researches and Trials In NeuroloGy-2) collaborators: Intravenous transplantation of mesenchymal stem cells preconditioned with early phase stroke serum: Current evidence and study protocol for a randomized trial. Trials 14: 317, 2013.

10. Chen JL, Li Y, Wang L, Lu M, Zhang XH and Chopp M: Therapeutic benefit of intracerebral transplantation of bone marrow stromal cells after cerebral ischemia in rats. J Neurol Sci 189: 49-57, 2001.

11. Wakabayashi K, Nagai A, Sheikh AM, Shiota Y, Narantuya D, Watanabe T, Masuda J, Kobayashi S, Kim SU and Yamaguchi S: Transplantation of human mesenchymal stem cells promotes functional improvement and increased expression of neurotrophic factors in a rat focal cerebral ischemia model. J Neurosci Res 88: 1017-1025, 2010.

12. Jiang Y, Jahagirdar BN, Reinhardt RL, Schwartz RE, Keene CD, Ortiz-Gonzalez XR, Reyes M, Lenvik T, Lund T, Blackstad M, et al: Pluripotency of mesenchymal stem cells derived from adult marrow. Nature 418: 41-49, 2002

13. Dvorakova J, Hruba A, Velebny V and Kubala L: Isolation and characterization of mesenchymal stem cell population entrapped in bone marrow collection sets. Cell Biol Int 32: 1116-1125, 2008.

14. Hayashida K, Sano M, Kamimura N, Yokota T, Suzuki M, Maekawa Y, Kawamura A, Abe T, Ohta S, Fukuda K and Hori S: $\mathrm{H} 2$ gas improves functional outcome after cardiac arrest to an extent comparable to therapeutic hypothermia in a rat model. J Am Heart Assoc 1: e003459, 2012.

15. Xu K, Puchowicz MA, Lust WD and LaManna JC: Adenosine treatment delays postischemic hippocampal CA1 loss after cardiac arrest and resuscitation in rats. Brain Res 1071: 208-217, 2006. 
16. Shen LH, Li Y, Chen J, Zhang J, Vanguri P, Borneman J and Chopp $\mathrm{M}$ : Intracarotid transplantation of bone marrow stromal cells increases axon-myelin remodeling after stroke. Neuroscience 137: 393-399, 2006.

17. Livak KJ and Schmittgen TD: Analysis of relative gene expression data using real-time quantitative PCR and the 2(-Delta Delta C(T)) method. Methods 25: 402-408, 2001

18. Lebouvier A, Poignard A, Cavet M, Amiaud J, Leotot J, Hernigou P, Rahmouni A, Bierling P, Layrolle P, Rouard H and Chevallier N: Development of a simple procedure for the treatment of femoral head osteonecrosis with intra-osseous injection of bone marrow mesenchymal stromal cells: Study of their biodistribution in the early time points after injection. Stem Cell Res Ther 6: 68, 2015.

19. Zheng W, Honmou O, Miyata K, Harada K, Suzuki J, Liu H, Houkin K, Hamada H and Kocsis JD: Therapeutic benefits of human mesenchymal stem cells derived from bone marrow after global cerebral ischemia. Brain Res 1310: 8-16, 2010.

20. Ohtaki H, Ylostalo JH, Foraker JE, Robinson AP, Reger RL, Shioda S and Prockop DJ: Stem/progenitor cells from bone marrow decrease neuronal death in global ischemia by modulation of inflammatory/immune responses. Proc Natl Acad Sci USA 105: 14638-14643, 2008.

21. Yiu G and He Z: Glial inhibition of CNS axon regeneration. Nat Rev Neurosci 7: 617-627, 2006.

22. Huber AB, Weinmann O, Brösamle C, Oertle T and Schwab ME: Patterns of Nogo mRNA and protein expression in the developing and adult rat and after CNS lesions. J Neurosci 22: 3553-3567, 2002.

23. Shen LH, Li Y, Chen J, Cui Y, Zhang C, Kapke A, Lu M, Savant-Bhonsale $\mathrm{S}$ and Chopp M: One-year follow-up after bone marrow stromal cell treatment in middle-aged female rats with stroke. Stroke 38: 2150-2156, 2007.

24. Shichinohe H, Ishihara T, Takahashi K, Tanaka Y, Miyamoto M, Yamauchi T, Saito H, Takemoto H, Houkin K and Kuroda S: Bone marrow stromal cells rescue ischemic brain by trophic effects and phenotypic change toward neural cells. Neurorehabil Neural Repair 29: 80-89, 2015

25. Nakano N, Nakai Y, Seo TB, Yamada Y, Ohno T, Yamanaka A, Nagai Y, Fukushima M, Suzuki Y, Nakatani T and Ide C: Characterization of conditioned medium of cultured bone marrow stromal cells. Neurosci Lett 483: 57-61, 2010.

26. Skoff RP, Bessert D, Barks JD and Silverstein FS: Plasticity of neurons and glia following neonatal hypoxic-ischemic brain injury in rats. Neurochem Res 32: 331-342, 2007.
27. Wang X, Deng J, Boyle DW, Zhong J and Lee WH: Potential Role of IGF-I in hypoxia tolerance using a rat hypoxic-ischemic model: Activation of hypoxia-inducible factor 1alpha. Pediatr Res 55: 385-394, 2004.

28. Lehtinen MK, Zappaterra MW, Chen X, Yang YJ, Hill AD, Lun M, Maynard T, Gonzalez D, Kim S, Ye P, et al: The cerebrospinal fluid provides a proliferative niche for neural progenitor cells. Neuron 69: 893-905, 2011.

29. Šerbedžija P, Madl JE and Ishii DN: Insulin and IGF-I prevent brain atrophy and DNA loss in diabetes. Brain Res 1303: 179-194, 2009.

30. Landi S, Ciucci F, Maffei L, Berardi N and Cenni MC: Setting the pace for retinal development: Environmental enrichment acts through insulin-like growth factor 1 and brain-derived neurotrophic factor. J Neurosci 29: 10809-10819, 2009.

31. Lopez-Lopez C, LeRoith D and Torres-Aleman I: Insulin-like growth factor I is required for vessel remodeling in the adult brain. Proc Natl Acad Sci USA 101: 9833-9838, 2004.

32. Guan J: Insulin-like growth factor-1 (IGF-1) derived neuropeptides, a novel strategy for the development of pharmaceuticals for managing ischemic brain injury. CNS Neurosci Ther 17: 250-255, 2011.

33. Ueki K, Fruman DA, Brachmann SM, Tseng YH, Cantley LC and Kahn CR: Molecular balance between the regulatory and catalytic subunits of phosphoinositide 3-kinase regulates cell signaling and survival. Mol Cell Biol 22: 965-977, 2002.

34. Khokhlatchev AV, Canagarajah B, Wilsbacher J, Robinson M, Atkinson M, Goldsmith E and Cobb MH: Phosphorylation of the MAP kinase ERK2 promotes its homodimerization and nuclear translocation. Cell 93: 605-615, 1998.

35. Peruzzi F, Prisco M, Dews M, Salomoni P, Grassilli E, Romano G, Calabretta B and Baserga R: Multiple signaling pathways of the insulin-like growth factor 1 receptor in protection from apoptosis. Mol Cell Biol 19: 7203-7215, 1999.

36. Scheid MP and Duronio V: Dissociation of cytokine-induced phosphorylation of Bad and activation of PKB/akt: Involvement of MEK upstream of Bad phosphorylation. Proc Natl Acad Sci USA 95: 7439-7444, 1998.

37. Tanaka R, Miyasaka Y, Yada K, Ohwada T and Kameya T: Basic fibroblast growth factor increases regional cerebral blood flow and reduces infarct size after experimental ischemia in a rat model. Stroke 26: 2154-2159, 1995. 